\title{
A TRYPANOSOMA FOUND IN BLOOD OF CATTLE IN INDIA.
}

By Captain Durrant M.R.C.V.S., A.V.D., and Captain J. D. E Holmes, M.A., M.R.C.V.S., C.V.D., INDIA.

A TRYPANOSOMA having the characters shown in the accompanying diagram (Plate IV.) was recently observed at the post-mortem of a bull used for experimental purposes at Muktesar Laboratory.

A small bull of Hill Breed, weighing about 250 lbs. and aged five years, was used with others for the purpose of testing the potency of a bulk of anti-rinderpest serum. This bull got a very slight attack of rinderpest accompanied by a loss of condition from which he appeared to be recovering. A week after the rinderpest reaction had passed over, and during which the bull fed wcll, other symptoms appeareddiarrhœa, intermittent fever, loss of appetite, staring coat, anæmia. The bull died six days later. From the appearance of the secondary symptoms we suspected the complication of piroplasmosis, and on two different occasions examined films of blood stained by a modification of Romanowsky's method. There were many signs of an anemic condition, the red corpuscles were pale, many with crenatcd edges, numerous poikilocytes, increased number of esinophiles, and many erythrocytes showing basophile granulations. The latter condition I have observed in almost every instance when examining blood from cattle recently recovered from rinperest. No piroplasmata were found.

At the post-mortem nothing further than a general anamic condition of the body was observed. On examining a fresh smear from the spleen, several living trypanosomata were seen in each field. At first it was suspected that the bull might have been inoculated with horse surra by means of the tabanidae which at this time were prevalent in large numbers. We had several experimental animals (ponies, rabbits, and guinea pigs) suffering from surra, and on several occasions had found the blood of these horse flies teeming with trypanosomata.

However, after staining some specimens taken from the spleen with double stain, it was seen that this trypanosoma varied in many respects from that of horse surra. It is 2 to $4 \mu$ shorter, the body is much thicker, and the posterior end blunter. The centrosome is situated much nearer to the nucleus, and not close to the posterior end as in the trypanosoma evansi. The nucleus and flagellum are more difficult to stain.

This trypanosoma appears to us to more closely resemble the form described by Theiler as affecting cattle in South Africa ${ }^{1}$.

Unfortunately, inoculation experiments were impossible owing to the decomposed condition of spleen and blood.

The observance of this trypanosoma has many points of interest. Up to the present it has not been demonstrated that the cattle of India suffer from trypanosomiasis. It is known that cattle experimentally inoculated with the trypanosoma evansi are not affected. After inoculation the trypanosoma appears in the blood in small numbers, at 
intervals, for two or three weeks, after which it entirely disappears, and the health of the animal is in no way affected.

It has also been found that the blood of a bull inoculated with horse surra, and from whose blood the trypanosoma has for many months been absent, is still capable of conveying the disease to other susceptible animals-horse, rabbit, guinea-pigs, etc. It has yet to be ascertained if the trypanosoma herewith described is capable of causing any grave affection among cattle, or whether, like the trypanosoma evansi, it simply lies dormant in the system. In the case under description there is no doubt that, after the normal resistant power of the cells had been reduced by the attack of rinderpest, the trypanosoma assisted in bringing about a fatal termination.

No clinical symptoms ascribable to trypanosomiasis have been recorded in India, but this is no argument against the existence of the disease.

Until recently it was not known that the cattle of this country were infected with piroplasma.

In 1902 Stockman ${ }^{1}$ demonstrated the pear-shaped bodies in the blood of cattle being used for rinderpest experiments. Since then further investigation has shown that a very large percentage of cattle are affected, and, as far as our present knowledge goes, piroplasmosis by itself causes no symptom of illness in Indian cattle, but rather plays a secondary part when the animal is attacked by another debilitating disease.

In the Veterinary Record, 30th August 1902, there is an extract from an article by Laveran and Nocard describing a very fatal outbreak of surra among the cattle of Mauritius. The outbreak was ascribed to surra introduced by cattle imported from India. It is very improbable that this outbreak was caused by the trypanosoma evansi, which has never been noticed in cattle of India except when experimentally inoculated.

\title{
DESCRIPTION OF PLATE IV.
}

Full grown and young forms of trypanosoma obtained from spleen of bull. Magnification 2500 . Drawn with oil imm. apochromatic $2 \mathrm{~mm}$. lens. Ocular No. 8 compensating. Enlarged by photography one and a half and redrawn.

\section{EVOLUTION OF THE TRYPANOSOMA EVANSI.}

\author{
By J. D. E. Holmes, M.A., M.R.C.V.S., Capt. I.C.V.D., \\ Assistant Bacteriologist, Muktesar, India.
}

ONE of the greatest obstacles to the furtherance of this study is the difficulty of following in a fresh specimen the various stages through which the parasite passes. Most observers agree that it is in coloured preparations only that the evolution of this hæmatozoon can be suitably followed. The many improvements in the methods of staining, principally that of Romanowsky, and the various modifications of his method, introduced by Laveran, Ziemann, Nocht, and others, have greatly facilitated an investigation of this kind. There 